\title{
Journal of Molecular Biomarkers \& Diagnosis
}

\section{Nuclear Receptor FXR: A Potential Therapeutic Target for the Treatment of Diseases with Impaired Urine Concentration}

Xiaoyan Zhang ${ }^{* 1,2}$ and Youfei Guan ${ }^{1,2}$

${ }^{1}$ Advanced Institute of Medical Sciences, Dalian Medical University, Dalian, Liaoning 116044, P.R China

${ }^{2}$ AstraZeneca-Shenzhen University Joint Institute of Nephrology, Shenzhen University Health Science Center, Shenzhen 518060, P.R China

*Corresponding author: Xiaoyan Zhang, Advanced Institute of Medical Sciences, Dalian Medical University, Dalian, P.R China, Tel: +86 41186110221 ; E-mail: wserien@163.com

Rec date: Mar 08, 2016, Acc date: Mar 19, 2016, Pub date: Mar 25, 2016

Copyright: (c) 2016 Zhang X, et al. This is an open-access article distributed under the terms of the Creative Commons Attribution License, which permits unrestricted use, distribution, and reproduction in any medium, provided the original author and source are credited.

\begin{abstract}
Farnesoid $X$ receptor (FXR), the bile acid-activated nuclear receptor and member of the nuclear receptor superfamily, plays a key role in bile acid, lipid and glucose homeostasis. FXR is abundantly expressed in the kidney, but its physiological function remains mostly unknown. Recent studies have demonstrated that FXR is expressed in renal collecting ducts where it directly regulates the transcription of the aquaporin 2 (AQP2) gene. FXR gene deficiency results in a polyuric phenotype in mice. These results highlight a novel mechanism for FXR in mediating renal urine concentration independent of the antidiuretic hormone (ADH) system. FXR may represent a potential therapeutic target for treating diseases with urine concentrating defect, including hepatorenal syndrome and nephrogenic diabetes insipitus.
\end{abstract}

Keywords: Nuclear receptor; Water homeostasis; FXR; Aquaporin; Bile acid

\section{Introduction}

FXR belongs to the superfamily of nuclear receptor transcription factor and was originally named on the basis of its weak activation by farnesol. Shortly after that, it was termed bile acid receptor since bile acids at physiological levels were found to be potent endogenous ligands for this nuclear receptor [1]. As the bile acid nuclear receptor, FXR regulates hepatoenteric circulation of bile acids through the bile acid transporter [2]. As a member of the metabolic nuclear receptors, it also regulates the expression of many target genes in lipid and glucose metabolism and participates in glucose and lipid homeostasis [3]. In the recent years, increasing evidence has demonstrated that FXR also takes part in biological processes of liver protection such as the liver detoxification and liver regeneration [4].

In addition to the liver and small intestine, FXR is highly expressed in the kidney. However, its physiological role remains mostly unknown. Activation of FXR has been shown to attenuate diabetic albuminuria and fibrosis [5]. Recently, it has been reported that FXR activation maintains endogenous glutathione homeostasis and protects the kidney in uninephrectomized mice from obesity-induced injury [6]. These findings suggest that FXR may serve as a new therapeutic target to treat chronic kidney disease including diabetic nephropathy. However, to date the physiological role of FXR in the kidney remains poorly understood although FXR is highly expressed in the kidney. In this mini-review, we will discuss recent findings regarding the role of FXR in renal physiology with a focus on urine concentration regulation.

\section{Intrarenal localization of FXR}

As the first step to determine the biological significance of FXR in the kidney, a few groups have examined FXR's intrarenal localization using immunohistochemical staining technique $[7,8]$. It has been demonstrated that FXR is ubiquitously expressed in the epithelial cells of all renal tubules including the proximal tubules, distal convoluted tubules and collecting ducts. In contrast, FXR expression is very low in renal glomeruli $[9,10]$. These findings suggest that FXR may play an important role in water and solute reabsorption.

\section{Effect of FXR activation and inactivation on urine concentration}

High expression levels of FXR in renal tubules suggest a critical role this nuclear receptor might play in renal physiology. To determine the role of FXR in the kidney, we have treated mice with the endogenous FXR agonist, chenodeoxycholic acid (CDCA) and found that activation of FXR resulted in a significant decrease in urine output7. This finding indicates that FXR activation may increase urine concentration capacity. In support, in FXR gene knockout mice, a significant increase of urine volume was observed [7]. Collectively, these observations demonstrate that FXR may play a critical role in the maintenance of water homeostasis.

\section{The role of FXR in renal AQP2 expression}

Water reabsorption in the kidney is influenced by the water permeability and the osmotic gradient. Water permeability in renal tubules is determined by the expression and apical localization of many aquaporins (AQPs) [11]. Among them, AQP2 is selectively expressed in the principal cells of the collecting ducts, where it controls water reabsorption in this renal tubule segment and determines final urine volume. Although only a small amount of water is reabsorbed by renal collecting ducts, many regulatory mechanisms are involved in the regulation of AQP2 expression. The most important one is the AVP-V2 receptor system.

Arginin vasopressin (AVP or ADH) is synthesized by the hypothalamus and represents the most important hormone in 
Page 2 of 3

regulating water reabsorption in kidney. AVP interacts with the V2 receptor distributed at the basolateral membrane of collecting duct principal cells and enhances water reabsorption by increasing AQP2 expression and apical targeting through the cAMP-PKA signaling pathway12; 13. We found that FXR activation increases, while FXR inactivation decreases, AQP2 expression and apical membrane localization in the collecting ducts. Therefore, FXR may help modulate urine volume via the regulation of AQP2 in the kidney.

\section{AQP2 is a direct target gene of FXR}

Both systemic and local mechanisms may be involved in the regulation of renal AQP2 expression. In mice treated with CDCA or deficient for FXR gene, no alteration of serum AVP concentrations and renal V2 receptor levels was observed, suggesting the change in urine volume may be the result of direct action of FXR in renal collecting ducts.

Previous studies on other nuclear receptors have demonstrated that AQP2 may be under the control of many nuclear receptors including glucocorticoid receptor (GR), aldosterone receptor (AR) or mineralocorticoid receptor (MR), liver $\mathrm{X}$ receptor beta (LXR $\beta)$, estrogen receptor alpha (ER), and peroxisome proliferator activated receptor gamma (PPAR) in renal collecting duct cells [12-17], further supporting the possibility that FXR may directly regulate AQP2 expression in this tubular segment.

As a matter of fact, it has been reported that using both bioinformatics and ChIP-Seq approaches a functional FXR and RXRa heterodimer binding site was found in AQP4 gene, implying the direct gene regulation of FXR on AQP4 and possibly on other AQP channels. A few recent studies have showed that small heterodimer partner (SHP), another nuclear receptor, is an immediate target gene of FXR, which might be also involved in the effect of FXR on water homeostasis regulation $[18,19]$.

Recently, our group has analyzed human and mouse AQP2 gene sequence and found a putative FXRE located in the promoter region of AQP2 gene. By using luciferase reporter and Chip assays, we further confirmed that activated FXR can bind to the FXRE site in the promoter of AQP2 gene, thereby significantly increasing AQP2 expression.

Taken together, FXR is highly expressed in the kidney, especially in the renal collecting ducts, where its activation increases water reabsorption through directly upregulating AQP2 gene transcription.

\section{Perspective}

Renal collecting ducts are critical in determining urine volume under the control of both systemic hormone AVP and locally acting factors. Defects in the V2 receptor signaling pathway result in a polyuria phenotype, a state called nephrogenic diabetes insipitus (NDI) [9]. Currently, no effective treatment is available for patients with NDI. The finding that FXR activation reduces urine output via increasing AQP2 expression raises a possibility that clinically used CDCA may be a potential therapeutic agent for the treatment of this disease.

In addition, in patients with end-stage liver disease, advanced cirrhosis and cholestasis, renal function is frequently impaired with fluid retention due to increased renal water and sodium reabsorption, a clinical disorder known as hepatorenal syndrome [20].
In these patients, normal enterohepatic circulation of bile acids is disrupted, resulting in a high level of plasma bile acid. The findings that FXR activation increases AQP2 expression, while FXR inactivation decreases renal AQP2 abundance, support the idea that collecting duct FXR activation by increased exposure to both filtered and circulating bile acids may be involved in reduced water excretion in patients with hepatorenal syndrome. It also implies that FXR may represent a potential target for developing novel drugs in the treatment of this severe disorder with a very poor prognosis.

\section{Acknowledgements}

This work was supported by the National Basic Research Program of China (973 Program) (2012CB517504), the National Natural Science Foundation of China (81570636), and Shenzhen Peacock Plan and JCYJ 20140418095735626.

\section{References}

1. Makishima M, Okamoto AY, Repa JJ, Tu H, Learned RM, et al. (1999) Identification of a nuclear receptor for bile acids. Science 284: 1362-1365.

2. Inagaki T, Choi M, Moschetta A, Peng L, Cummins CL, et al. (2005) Fibroblast growth factor 15 functions as an enterohepatic signal to regulate bile acid homeostasis. Cell Metab 2: 217-225.

3. Thomas C, Pellicciari R, Pruzanski M, Auwerx J, Schoonjans K (2008) Targeting bile-acid signalling for metabolic diseases. Nat Rev Drug Discov 7: 678-693.

4. Wang C, Zhang F, Wang L, Zhang Y, Li X, et al. (2013) Poly(ADP-ribose) polymerase 1 promotes oxidative-stress-induced liver cell death via suppressing farnesoid X receptor alpha. Mol Cell Biol 33: 4492-4503.

5. Wang XX, Jiang T, Shen Y, Caldas Y, Miyazaki-Anzai S, et al. (2010) Diabetic nephropathy is accelerated by farnesoid $X$ receptor deficiency and inhibited by farnesoid $\mathrm{X}$ receptor activation in a type 1 diabetes model. Diabetes 59: 2916-2927.

6. Gai Z, Gui T, Hiller C, Kullak-Ublick GA (2016) Farnesoid X Receptor Protects against Kidney Injury in Uninephrectomized Obese Mice. J Biol Chem 291: 2397-2411.

7. Zhang X, Huang S, Gao M, Liu J, Jia X, et al. (2014) Farnesoid X receptor (FXR) gene deficiency impairs urine concentration in mice. Proc Natl Acad Sci USA 111: 2277-2282.

8. Higashiyama H, Kinoshita M, Asano S (2008) Immunolocalization of farnesoid X receptor (FXR) in mouse tissues using tissue microarray. Acta Histochem 110: 86-93.

9. Jiang T, Wang XX, Scherzer P, Wilson P, Tallman J, et al. (2007) Farnesoid $\mathrm{X}$ receptor modulates renal lipid metabolism, fibrosis, and diabetic nephropathy. Diabetes 56: 2485-2493.

10. Levi M (2011) Nuclear receptors in renal disease. Biochim Biophys Acta 181: 1061-1067.

11. Verkman AS (2012) Aquaporins in clinical medicine. Annu Rev Med 63: 303-316.

12. Moeller HB, Ritting S, Fenton RA (2013) Nephrogenic diabetes insipidus: essential insights into the molecular background and potential therapies for treatment. Endocr Rev 34: 278-301.

13. Sasaki S (2012) Aquaporin 2: from its discovery to molecular structure and medical implications. Mol Aspects Med 33: 535-546.

14. He Y, Gong L, Fang Y, Zhan Q, Liu HX, et al. (2013) The role of retinoic acid in hepatic lipid homeostasis defined by genomic binding and transcriptome profiling. BMC Genomics 14: 575.

15. Guan Y, Hao C, Cha DR, Rao R, Lu W, et al. (2005) Thiazolidinediones expand body fluid volume through PPARgamma stimulation of ENaCmediated renal salt absorption. Nat Med 11: 861-866.

16. Gabbi C, Kong X, Suzuki H, Hyun-Jin Kim, Gao M, et al. (2012) Central diabetes insipidus associated with impaired renal aquaporin-1 expression 
Citation: Zhang and Guan (2016) Nuclear Receptor FXR: A Potential Therapeutic Target for the Treatment of Diseases with Impaired Urine Concentration . J Mol Biomark Diagn 7: 100278. doi:10.4172/2155-9929.1000278

Page 3 of 3

in mice lacking liver X receptor beta. Proc Natl Acad Sci USA 109 3030-3034.

17. Cheema MU, Irsik DL, Wang Y, Miller-Little W, Hyndman KA (2015) Estradiol regulates AQP2 expression in the collecting duct: a novel inhibitory role for estrogen receptor alpha. Am J Physiol Renal Physiol 309: 305-317.

18. Hu Z, Ren L, Wang C, Liu B, Song G (2012) Effect of chenodeoxycholic acid on fibrosis, inflammation and oxidative stress in kidney in highfructose-fed Wistar rats. Kidney Blood Press Res 36: 85-97.
19. Proctor G, Jiang T, Iwahashi M, Wang Z, Li J, et al. (2006) Regulation of renal fatty acid and cholesterol metabolism, inflammation, and fibrosis in Akita and OVE26 mice with type 1 diabetes. Diabetes 55: 2502-9.

20. Baraldi O, valentine C, Donati G, Comai G, Cuna V, et al. (2015) Hepatorenal syndrome: Update on diagnosis and treatment. World $\mathrm{J}$ Nephro 6: 511-20. 